\title{
Research on information updating of enterprise members based on learning network
}

\author{
Wu Xia ${ }^{1, a,{ }^{*}}$ \\ School of economics and management, Jiangsu University of Science and Technology, Zhenjiang, \\ Jiangsu, China \\ a383734304@qq.com \\ *Corresponding author
}

Keywords: learning network; social impact model; consensus; leadership independence

\begin{abstract}
Based on the enterprise learning behavior between members of the establishment of learning network, the use of social influence model analysis showed that the convergence and consensus learning network, according to the two situations: enterprise leadership and dogmatic open enterprise, an empirical test on a number of updates, two kinds of information will eventually converge to a the value of the enterprise, the convergence speed of the open enterprise information faster convergence than authoritarian leadership.
\end{abstract}

\section{基于学习网络的企业成员信息更新研究 \\ 伍霞 $1, \mathrm{a},{ }^{*}$ \\ 江苏科技大学经济管理学院, 镇江, 江苏, 中国 \\ a383734304@qq.com \\ *通讯作者}

关键词: 学习网络;社会影响模型;共识;领导独断

中文摘要. 本文以企业成员之间的学习行为建立学习网络, 利用社会影响模型分析发现学习 网络的收玫性和共识性, 根据两种现实情况一一开放式的企业和领导独断式的企业, 实证检 验了在多次的更新下, 两种情况信息最终都会收玫到一个值, 开放式的企业收敛速度比领导 独断的企业收敛信息较快。

\section{1. 引言}

企业成员通常进行相互沟通、观察和学习这样就形成了社会网络，这种社会网络也是一 种学习网络, 成员之间交换信息, 并且不断更新信息, 来完成工作上的一些信息更新和意见 统一。现有的研究发现, 成员之间的信息在交流的过程具有观察学习的特征。学者 DeGroot ${ }^{[1]}$ 创建了信息传播、观点形成和共识达成的网络交互作用的开创性模型，也就是模仿和社会影 响模型, 它是更充分地理解学习网络如何影响信息传播和意见形成理论的一个非常简单而合 适的起点。国内学者广雄用德格鲁特模型研究社会学习的价格预期, 在三种情况下价格具有 收敛性和共时性 ${ }^{[2]}$ 。Bolton et al.(2009) ${ }^{[3]}$, Pena et al.(2005) ${ }^{[4]}$ 通过实验发现, 当判断者信息不 足或者市场噪声太大时, 判断者会观察学习其他判断者的行为并进行模仿。加之判断者都是 有限理性的, 自身掌握的信息并不全面, 在这样的情况下, 判断者很可能根据别人在类似的 情况下做出的决定来做决定, 于是形成了羊群效应, 成员的信息最终统一。羊群效应在股票 投资和市场营销等领域随处可见, 不少学者已经做了大量研究, 但是在企业学习网络中的研 究还不多。 
通过文献梳理发现，关于企业成员之间的学习行为国外已有一些研究，但是国内较少， 而且针对成员之间具体如何学习、向谁学习、最终有怎样的学习结果等这些问题还需要更进 一步的研究。本文通过验证企业成员之间的学习网络更新如何收玫, 建立了两种学习不同学 习矩阵，实证检验了信息更新的不同之处。

\section{2. 企业学习网络}

企业成员就是网络中的节点, 成员之间通过学习建立关系, 假设在一个学习网络中有 $\mathrm{n}$ 个成员, 学习网络可以看做是一个有向加权网络, 成员和成员之间的连接存在着方向, 也就 是在学习的时候成员向谁学习。加权网络的意思是, 对学习的对象的重要程度进行区分, 总 有一些比较重要的学习对象和不太重要的学习对象, 用权值来衡量一个学习对象的重要程度。 把 $a_{i, j} \geq 0$ 作为成员 $\mathrm{i}$ 向成员 $\mathrm{j}$ 的学习程度, 也就是成员 $\mathrm{j}$ 对成员 $\mathrm{i}$ 的影响程度, $a_{i}=\left(a_{i, 1}, a_{i, 2}, \ldots, a_{i, n}\right)$ 表示个体 $\mathrm{i}$ 对成员学习程度的主观权重向量, 且有 $\sum_{j \in n} a_{i, j}=1$ 。网络每个 个体的主观权值向量组成的社会权重矩阵为:

$$
T=\left[\begin{array}{c}
a_{1} \\
\vdots \\
a_{n}
\end{array}\right]=\left[\begin{array}{ccc}
a_{1,1} & \cdots & a_{1, n} \\
\vdots & \vdots & \vdots \\
a_{n, 1} & \cdots & a_{n, n}
\end{array}\right]
$$

且设定如果 $\mathrm{i}$ 征询来自 $\mathrm{n}$ 个不同行为人的意见, 其中包括 $\mathrm{j}$, 则 $a_{i j}=1 / n$ 。

如图图 1 是一个由 3 个成员组成的简单的学习网络, 其中成员 1 向成员 $2 、 3$ 学习, 学习 的分配权重都是 $1 / 2$, 成员 2 向成员 1 和自己学习, 学习的分配权重都是 $1 / 2$, 成员 3 向成员 2 完全学习。

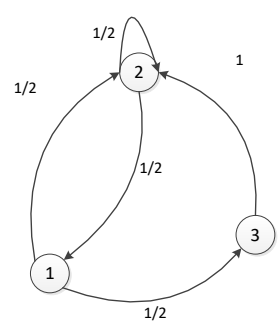

图 1 学习网络

\section{3. 不断更新信息的学习网络}

\section{1 信息更新}

在 $\mathrm{n}$ 个行为人的学习网络中, 成员对事物都有一个最初的观点, 由 $\mathrm{n}$ 维概率向量 $P=\left(P_{1}, P_{2}, \ldots, P_{n}\right)$ 来表示。每个 $P_{i}$ 都位于区间 $[0,1]$ 之内, 在企业学习网络中表示个人参加到特 定活动中的可能性概率。网络中每个成员 $\mathrm{i}$ 在初始时间看到某事物的一个受干扰的信号 $P_{i}(0)=\mu+\varepsilon_{i}, \varepsilon_{i} \in R$ 为个体 $\mathrm{i}$ 获得信息的随机干扰项。个体 $\mathrm{i}$ 并不一定相信自己获得的信息， 而是会认真听取邻居和朋友等人的意见, 学习的模式是通过可能的权重和有向的 $n \times n$ 非负矩 阵 $\mathrm{T}$ 来体现。信息是随着时间的发展而更新的, 所以

$$
P(t)=T P(t-1)=T^{t} P
$$

每个时刻开始时, 成员从邻居那里获得他们的信息, 然后根据邻居对个体的影响大小进 行线性加权平均, 然后形成自己新的信息

$$
\mu_{i, t}(\theta)=\sum a_{i, j} \mu_{j, t}(\theta)
$$




\section{2 信息更新的收敛性和共识}

模型中, 成员的观点会影响到他人下一期的观点, 而这种影响又会随着下一期他人对自 己观点的影响融入到自己再下一期的观点之中，观点在人们的模仿和相互影响下互相交织， 形成社会在某一时刻的观点状态。如果社会影响矩阵 $\mathrm{T}$ 是收玫的, 即对于所有最初信仰向量 $\mathrm{P}$ 而言, 极限 $\lim _{t} T^{t}$ 存在, 则社会观点在互相交流达到某一时间段后, $\mathrm{P}$ 就处于一个稳定状态, 不再进一步随着时间发生改变, 社会中每个人的观点锁定在某一特定值, 观点就达到了收玫 状态。社会权重矩阵 $\mathrm{T}$ 是收玫的, 当且仅当每个强连通且闭的节点集是无周期的。其中, 有 向环存在于从 $\mathrm{i}$ 到 $\mathrm{j}$ 存在一条有向连结当且仅当 $T_{i j}>0$ 的有向网络中, $\mathrm{T}$ 是无周期性的是指随 意有向环长度的最大公因式是 1 。

\section{3 领导独断的影响矩阵}

在共识的基础上, 下面研究学习网络中成员的社会影响。成员的社会影响力即社会网络 中的每个行为人对于极限收玫观点的形成具有多大的影响, 如果某个个体对观点的最终影响 权重较大, 则此个体可看作是在某观点上意见的领导者。

令 $P(0)$ 是一个任意的起始信仰向量, 而 $P(\infty)=\left(P^{\infty}, \cdots, P^{\infty}\right)$ 是极限共识信仰向量。为了寻 求每个行为人具有的极限影响, 要有向量 $s \in[0,1]^{n}$ 使得 $\sum_{i} s_{i}=1$ 并且 $P^{\infty}=s \cdot P(0)=\sum_{i} s_{i} P_{i}(0)$ 。

由于以 $P(0)$ 或 $P(1)=T P(0)$ 的起点产生了相同的极限, 所以必然有 $s \cdot P(1)=s \cdot P(0)$, 因 此

$$
s \cdot(T P(0))=s \cdot P(0),
$$

这一等式必须对每个 $P(0)$ 都成立, 由此可得

$$
s T=s
$$

\section{4. 不同网络结构的企业模式}

\section{1 领导独断式}

在企业学习网络中, 领导者占据着重要的社会影响。当具有领导性地位是高度的独断者, 其对某件事的预测不受其他成员的意见影响, 并且对其他成员进行积极地引导。此结构的社 会影响矩阵形式如下:

$$
T_{3}=\left[\begin{array}{cccc}
1 & 0 & \cdots & 0 \\
a_{21} & a_{22} & \cdots & a_{2 n} \\
\vdots & \vdots & \vdots & \vdots \\
a_{n, 1} & a_{n, 2} & \cdots & a_{n, n}
\end{array}\right]
$$

此结构下，信息流通存在一个强连通且闭的节点集是：\{1\}。领导不受外界信息的干扰和 影响，其他成员给领导的意见赋予较大的权重，领导在整个社会网络信息交流中处于不可动 摇的核心地位，这种结构在一个有较高社会影响且独断的领导人的公司是存在的。

\section{2 全开放式}

这种情况下, 也就是一般情况，领导视为普通成员，大家都达到一个平等的地位，没有 领导和下属之分, 大家相互学习。此时的社会影响矩阵为一般情况, 形式为 


$$
T=\left[\begin{array}{ccc}
a_{1,1} & \cdots & a_{1, n} \\
\vdots & \vdots & \vdots \\
a_{n, 1} & \cdots & a_{n, n}
\end{array}\right]
$$

此结构下，每个成员的意见都可以通过网络传播直接或间接地影响到另一成员，每个成 员对最后的信息形成都具有一定的影响, 影响程度取决于其他成员依据成员的身份赋予他的 影响权重。

\section{5. 实证分析}

本文选取较小规模的某个软件开发公司管理层作为实证对象, 大家平常在技术和信息上 存在频繁交流, 而且管理层不同部门人员会更频繁跨部门交流, 所以学习网络特征比较明显。 搜集了对公司对于两次自愿活动参加的意愿及学习情况。所得的到的有向网络表示在图三中。 其中 1 为总领导人， $2 、 3 、 4$ 为部门主管，5、6、7、8、9 为普通成员，且 $2 、 5 、 6$ 为同一部 门, $3 、 6$ 为同一部门, $4 、 8 、 9$ 为同一部门。

\section{1 全开放式学习}

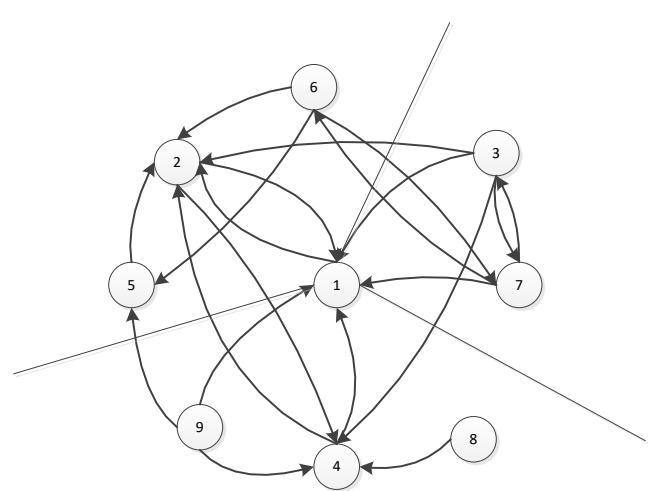

图 2 某企业小型研发组织学习观察网络

在第一次活动中, 大家是全开放式学习模式, 如图 2。则此网络中的学习矩阵为

成员每个人的最初信息为

$$
T=\left[\begin{array}{ccccccccc}
1 / 2 & 1 / 2 & 0 & 0 & 0 & 0 & 0 & 0 & 0 \\
1 / 3 & 1 / 3 & 0 & 1 / 3 & 0 & 0 & 0 & 0 & 0 \\
1 / 5 & 1 / 5 & 1 / 5 & 1 / 5 & 0 & 0 & 1 / 5 & 0 & 0 \\
1 / 3 & 1 / 3 & 0 & 1 / 3 & 0 & 0 & 0 & 0 & 0 \\
0 & 1 / 2 & 0 & 0 & 1 / 2 & 0 & 0 & 0 & 0 \\
0 & 1 / 4 & 0 & 0 & 1 / 4 & 1 / 4 & 1 / 4 & 0 & 0 \\
1 / 4 & 0 & 1 / 4 & 0 & 0 & 1 / 4 & 1 / 4 & 0 & 0 \\
0 & 0 & 0 & 1 / 2 & 0 & 0 & 0 & 1 / 2 & 0 \\
1 / 4 & 0 & 0 & 0 & 1 / 4 & 1 / 4 & 0 & 0 & 1 / 4
\end{array}\right],
$$

$$
P=\left(\begin{array}{lllllllll}
0.8 & 0.6 & 0.55 & 0.4 & 0.6 & 0.7 & 0.4 & 0.65 & 0.7
\end{array}\right) 。
$$

本文意愿值设定, $\mathrm{P}$ 小于 0.5 , 则为不参与, $\mathrm{P}$ 大于等于 0.5 则为参与。 通过计算得到 


$$
T^{t} \rightarrow\left[\begin{array}{lllllllll}
0.4 & 0.4 & 0 & 0.2 & 0 & 0 & 0 & 0 & 0 \\
0.4 & 0.4 & 0 & 0.2 & 0 & 0 & 0 & 0 & 0 \\
0.4 & 0.4 & 0 & 0.2 & 0 & 0 & 0 & 0 & 0 \\
0.4 & 0.4 & 0 & 0.2 & 0 & 0 & 0 & 0 & 0 \\
0.4 & 0.4 & 0 & 0.2 & 0 & 0 & 0 & 0 & 0 \\
0.4 & 0.4 & 0 & 0.2 & 0 & 0 & 0 & 0 & 0 \\
0.4 & 0.4 & 0 & 0.2 & 0 & 0 & 0 & 0 & 0 \\
0.4 & 0.4 & 0 & 0.2 & 0 & 0 & 0 & 0 & 0 \\
0.4 & 0.4 & 0 & 0.2 & 0 & 0 & 0 & 0 & 0
\end{array}\right]
$$

由 $s T=s$, 得 $s=(0.4,0.4,0,0.2,0,0,0,0,0)$, 即得到网络中的各成员的社会影响力, 且收 敛速度为 13 。

最终信息收敛 $P(\infty)=(0.64,0.64,0.64,0.64,0.64,0.64,0.64,0.64,0.64)$ 。网络中成员信息随 时间变化如图 3。

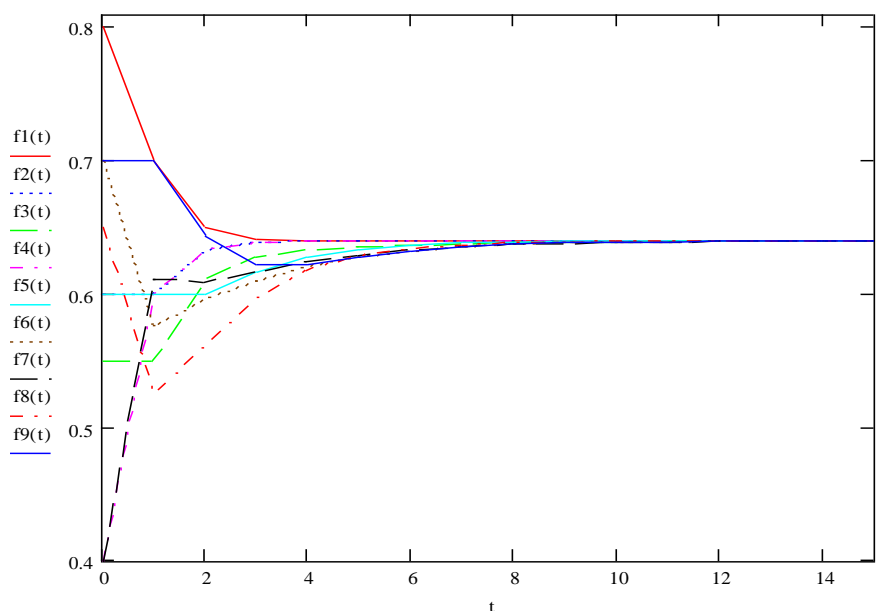

图 3 个人信息随时间演化图

从结果和演化图得到, 此学习网络中, 成员 1 和成员 2 的社会影响力最大, 都为 0.4 , 成 员 4 也具有一定的影响力, 说明在最后的学习中，成员基本都是向成员 $1 、 2 、 4$ 学习，进行 信息更新。最后更新得到的信息，也与这三位成员的最初信息有关，不管其他成员最初信息 是多少。通过 15 次的更新, 最终成员达成一致的, 都参与活动。

\section{2 领导独断的学习}

在第二次活动中，学习网络是领导独断的学习模式，如图 4,

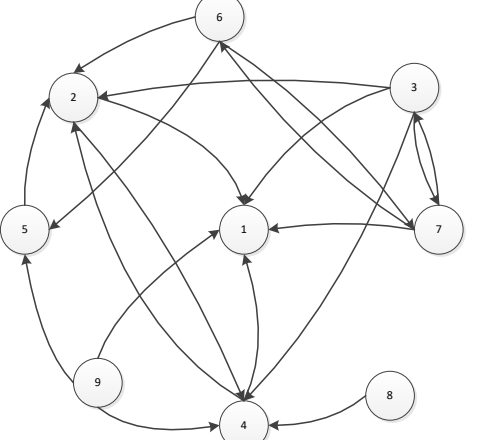

图 4 领导独断的学习观察网络

则此网络中的学习矩阵为 


$$
T=\left[\begin{array}{ccccccccc}
1 & 0 & 0 & 0 & 0 & 0 & 0 & 0 & 0 \\
1 / 3 & 1 / 3 & 0 & 1 / 3 & 0 & 0 & 0 & 0 & 0 \\
1 / 5 & 1 / 5 & 1 / 5 & 1 / 5 & 0 & 0 & 1 / 5 & 0 & 0 \\
1 / 3 & 1 / 3 & 0 & 1 / 3 & 0 & 0 & 0 & 0 & 0 \\
0 & 1 / 2 & 0 & 0 & 1 / 2 & 0 & 0 & 0 & 0 \\
0 & 1 / 4 & 0 & 0 & 1 / 4 & 1 / 4 & 1 / 4 & 0 & 0 \\
1 / 4 & 0 & 1 / 4 & 0 & 0 & 1 / 4 & 1 / 4 & 0 & 0 \\
0 & 0 & 0 & 1 / 2 & 0 & 0 & 0 & 1 / 2 & 0 \\
1 / 4 & 0 & 0 & 0 & 1 / 4 & 1 / 4 & 0 & 0 & 1 / 4
\end{array}\right],
$$

成员每个人的最初信息为

$$
P=\left(\begin{array}{lllllllll}
0.8 & 0.6 & 0.55 & 0.4 & 0.6 & 0.7 & 0.4 & 0.65 & 0.7
\end{array}\right) \text { 。 }
$$

通过计算得到 $s=(1,0,0,0,0,0,0,0,0)$, 收敛速度为 20 。网络中成员信息随时间变化如图 5 。

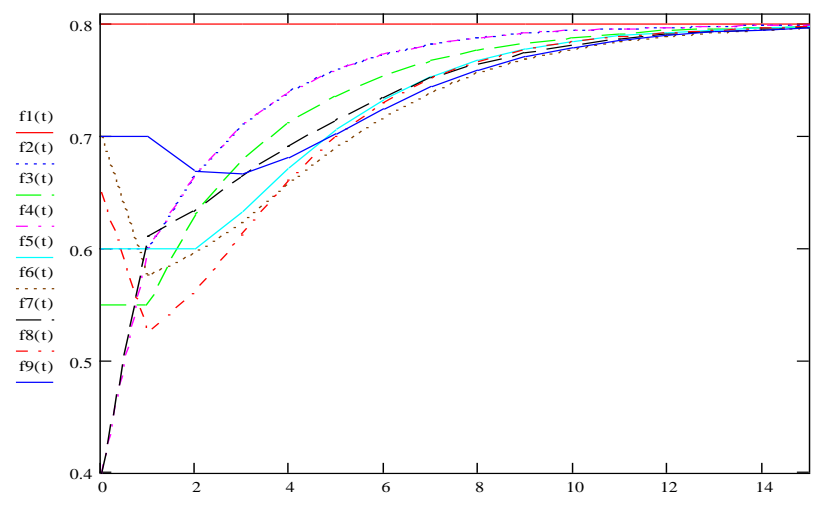

图 5 个人信息随时间演化图

从结果和演化图得到, 此学习网络中，由于成员 1 是独断的，且其他成员向他学习，所 以他的影响最大, 最后更新得到的信息, 只与成员 1 的最初信息有关, 不管其他成员最初信 息是多少。通过 20 次的更新, 最终成员达成一致, 都参与活动。与开放式学习相比, 达成一 致所需要的时间更长远, 但是最终收敛的意愿较高。

\section{6. 结论}

学习网络对企业管理预期具有重要影响, 利用好企业之间的学习网络能很好的帮助管理 者达到预期管理效果。本文利用德格鲁特的社会影响模型, 依据网络学习特征, 把社会主体 分为领导和普通成员两个相互作用的种群，通过这些主体间的不同影响程度，探讨了两种社 会影响结构矩阵下学习网络的收敛性、共识性、收敛速度和各主体的影响力程度。通过模拟 研究, 得出以下结论:

(1) 无论是独断的领导学习结构, 还是全开放的学习结构, 最终个人信息都能够收玫并 最终达成共识。

（2）信息收敛都需要一定的时间，独断的领导学习结构下，收敛的速度较慢，全开放的 社会影响结构下收敛的速度较快。

(3) 最终信息收敛结果只与有社会影响的成员最初信息有关，社会影响为 0 的成员的最 初信息不影响最终的收敛结果，但是在信息变化的过程还是具有一定影响。

通过模拟分析, 本文认为领导要想发挥学习网络的有效性, 一方面要推进各主体之间信 息交互的广度和速度以加快预期收玫的速度，另一方面还要努力提高领导的影响力, 这样有 助于领导达到自己预期的结果。 


\section{References}

[1]. Pavlou P A, Gefen D. Psychological violation in online marketplace: anlecedents, consequences, and moderating role [J]. Information Systems Research, 2005, 16(4):372-399.

[2]. Kuang Xiong.The Formation Mechanism of Social Expectation Based on the DeGroot Model and the Effectiveness of the Center Bank's Expectation Guidance[J]. Management Review, 2016,28(07):200-212.

[3]. Pereira J, Takahashi K, Ahumada L. Flexibility dimensions to control the bullwhip effect in a supply chain [J]. International Journal of Production Research, 2009,47 (22):6357-6374.

[4]. Steinel W, De Dreu C. Social motives and strategic misrepresentation in social decision making [J]. Journal of Personality and Social Psychology, 2004, (86):419-434. 